\title{
Lattice Boltzmann simulation of droplet generation in a microfluidic cross-junction
}

\author{
Haihu Liu and Yonghao Zhang* \\ Department of Mechanical Engineering, University of Strathclyde, Glasgow G1 1XJ, \\ UK
}

\begin{abstract}
Using the lattice Boltzmann multiphase model, numerical simulations have been performed to understand the dynamics of droplet formation in a microfluidic cross-junction. The influence of capillary number, flow rate ratio, viscosity ratio, and viscosity of the continuous phase on droplet formation has been systematically studied over a wide range of capillary numbers. Two different regimes, namely the squeezinglike regime and the dripping regime, are clearly identified with the transition occurring at a critical capillary number $C a_{c r}$. Generally, large flow rate ratio is expected to produce big droplets, while increasing capillary number will reduce droplet size. In the squeezing-like regime $\left(C a \leq C a_{c r}\right)$, droplet breakup process is dominated by the squeezing pressure and the viscous force; while in the dripping regime $\left(C a>C a_{c r}\right)$, the viscous force is dominant and the droplet size becomes independent of the flow rate ratio as the capillary number increases. In addition, the droplet size weakly depends on the viscosity ratio in both regimes and decreases when the viscosity of the continuous phase increases. Finally, a scaling law is established to predict the droplet size.
\end{abstract}

Key words: droplet generation, microdroplet technology, lattice Boltzmann method, multiphase flow

\section{Introduction}

Rapid development of microfabrication technologies has facilitated a broad range of microfluidic applications especially in biology and chemistry. Microdroplet technology has recently emerged as a promising flexible platform for microfluidic functions [1-3] where a droplet acts as an individual chemical reactor. As samples/reagents are confined in the droplets, it can avoid sample/surface interaction and thus eliminate surface adsorption and cross sample contamination. The miniaturization of the entire process can enable the rapid analysis of very small quantities of droplet samples in a portable, automated and inexpensive format [4]. Many microfluidic devices have emerged to generate uniform

*Corresponding author. Email address: yonghao.zhang@strath.ac.uk (Y. Zhang) 
droplets, including geometry-dominated devices [5, 6], flow-focusing devices [7-10], Tjunctions [11-16] and co-flowing devices $[17,18]$.

Although flow physics of droplet generation at T-junctions has been extensively investigated both experimentally and numerically, significant effort is required to understand droplet generation in a confined cross-junction. The droplet dynamics in a microfluidic cross-junction is very complicated. Many coupled factors will affect the droplet formation process, e.g. interfacial tension, wetting properties and confinement of flow channels, fluid flow rates and viscosities. Cubaud et al. [8] investigated the liquid/gas flows in a cross-junction and found that the bubble breakup could be understood as the competition between the pressure drops in the liquid and gas phases. The bubble size could be predicted by the gas/liquid flow rate ratio. Garstecki et al. [9] investigated the mechanism for bubble breakup process in the cross-junction with a small orifice, and observed that the collapsing rate of the neck is quasi-stationary and proportional to the liquid flow rate. Tan et al. [19] studied the formation mechanism of plug flow in an oil/water microfluidic cross-junction. They found that the plug size depends on the flow rate ratio of both fluids and the capillary number. Recently, Fu et al. [10] found that the bubble (slug) breakup process in a cross-junction is mainly controlled by the collapse stage, during which the collapse rate of the thread neck and the collapse time were affected by the gas/liquid flow rate ratio and the viscosity of the liquid phase. Although the experimental studies have helped to understand the underlying physics, the current available data are sporadic. Various materials were used to fabricate the microchannels with a diverse range of dimensions, and the experiments are operated under a wide range of flow conditions with different fluids. Consequently, the information is fragmented, which leads to inconclusive and even incompatible findings. On the other hand, experiments at such small scale are still difficult. For example, it is challenging to accurately measure droplet size, pressure and velocity fields, and droplet deformation, breakup, and coalescence.

Numerical study can be complementary to experimental investigation. Davidson et al. [20] used volume of fluid (VOF) method to predict the dynamics of droplet formation in an axisymmetric microfluidic flow-focusing geometry. The ending pinching and capillary-wave instability were found to be important for droplet breakup from the liquid jet with high flow rate. Hua et al. [18] used front-tracking/finite volume method to investigate the mechanism of droplet formation in a coflowing microchannel. The effects of the continuous phase flow rate, viscosity, and the interfacial tension on the droplet size were investigated in both dripping and jetting regimes, where the correlations of droplet size and Reynolds number, Weber number, capillary number, and viscosity ratio were obtained respectively. The lattice Boltzmann method (LBM) has shown great potential to model the interfacial interactions while incorporates fluid flow as a system feature [21]. It is a pseudo-molecular method tracking evolution of the distribution function of an assembly of molecules and built upon microscopic models and mesoscopic kinetic equations [22]. Its mesoscopic nature can provide many advantages of molecular dynamics, making the LBM especially useful for simulation of droplet dynamics [15,23-28]. 
Here, we apply a multiphase lattice Boltzmann model, using the phase-field theory to describe interfacial interactions, to investigate the droplet formation in a microfluidic cross-junction.

\section{Numerical Method}

A unifying feature of all the phase-field models is the existence of a free-energy functional, which not only determines the equilibrium properties, but also strongly influences the dynamics of a multiphase system. In these models, the evolution and breakup of interface emerge naturally which can be described by the thermodynamic theory such as the Cahn-Hilliard equation [29]. Due to its rich physical basis, the phase-field model has been widely used to simulate droplet deformation, coalescence, and breakup [30-33].

\subsection{Modelling two-phase immiscible flow}

In an incompressible two-phase system such as oil and water with densities of $\rho_{o}$ and $\rho_{w}$ respectively, the thermodynamics can be described by the Ginzburg-Landau free energy functional [?]:

$$
F(\rho, \phi, \nabla \phi)=\int\left[\Psi(\phi)+\frac{1}{2} \kappa|\nabla \phi|^{2}+\rho c_{s}^{2} \ln \rho\right] d V,
$$

where the total density $\rho$ is $\rho_{w}+\rho_{o}$; the order parameter $\phi$ describes the normalized difference in density of two fluids, i.e. $\phi=\left(\rho_{w}-\rho_{o}\right) / \rho ; \Psi(\phi)$ is the bulk free energy density for a homogeneous system, which can be chosen as a double-well form $\Psi(\phi)=\frac{1}{4} a\left(\phi^{2}-1\right)^{2}$, with $a$ being a positive constant; the term $\frac{1}{2} \kappa|\nabla \phi|^{2}$ denotes the free energy excess in the interfacial region, which is defined as the interface energy between different phases with $\kappa$ relating to the interfacial tension; $c_{s}$ is the lattice speed of sound. Note that the final term in the free energy functional does not affect the phase behavior, which is introduced to enforce incompressibility in LBM.

The chemical potential $\mu$ is defined as the variational derivative of the free energy functional with respect to the order parameter, i.e.,

$$
\mu=\delta F / \delta \phi=\Psi^{\prime}(\phi)-\kappa \nabla^{2} \phi=a \phi\left(\phi^{2}-1\right)-\kappa \nabla^{2} \phi .
$$

The equilibrium interface profile can be obtained from Eq. (2.2) at $\mu=0$, which leads to two stable uniform solutions $\phi= \pm 1$ representing the coexisting bulk phases.

For a planar oil-water interface in a quiescent fluid for an infinite system, the order parameter profile across the interface can be given by

$$
\phi(z)=\tanh (z / \xi),
$$

where $z$ is the spatial location normal to the interface $(z=0)$, and $\xi$ is a parameter proportional to the interface thickness, which is defined as

$$
\xi=\sqrt{2 \kappa / a} \text {. }
$$


Since the interfacial tension $\sigma$ can be interpreted as the excess free energy per unit interface area, for a plane interface in equilibrium, it can be evaluated by [34]

$$
\sigma=\int_{-\infty}^{+\infty} \kappa\left(\frac{\mathrm{d} \phi}{\mathrm{d} z}\right)^{2} d z
$$

From Eqs.(2.3)-(2.5), we can get

$$
\sigma=\frac{4 \kappa}{3 \xi}
$$

Equations (2.4) and (2.6) suggest that we can determine the parameters $\kappa$ and $a$ by specifying the interfacial tension and the interface thickness.

The hydrodynamics of a binary fluid can be described by the Navier-Stokes and Cahn-Hilliard equations [35]:

$$
\begin{gathered}
\nabla \cdot \vec{u}=0, \\
\rho\left(\partial_{t} \vec{u}+\vec{u} \cdot \nabla \vec{u}\right)=-\nabla \cdot \boldsymbol{P}+\nabla \cdot\left[\eta\left(\nabla \vec{u}+\nabla \vec{u}^{\mathrm{T}}\right)\right], \\
\partial_{t} \phi+\vec{u} \cdot \nabla \phi=\nabla \cdot(M \nabla \mu),
\end{gathered}
$$

where $\vec{u}$ is the fluid velocity, $\eta$ is the dynamic viscosity, and $M$ is the Cahn-Hilliard mobility. The equilibrium properties of the fluid are involved in the equations of motion through the chemical potential and the pressure tensor $\boldsymbol{P}$, which can be derived via the Gibbs-Duhem relation [35]:

$$
\nabla \cdot \boldsymbol{P}=\nabla\left(\rho c_{s}^{2}\right)+\phi \nabla \mu .
$$

When the fluid-solid interaction is considered, the chemical potential at the solid wall is difficult to be specified for evaluation of $\nabla \mu$ at the fluid lattice sites next to the wall. To mitigate this problem, we reform Eq.(2.10) as

$$
\nabla \cdot P=\nabla p-\mu \nabla \phi,
$$

where $p=\rho c_{s}^{2}+\phi \mu$ is the modified pressure. Once the pressure tensor is expressed as Eq.(2.11) in the Navier-Stokes equations, $p$ can be simply incorporated in the modified equilibrium distribution function and the interfacial force term $\mu \nabla \phi$ can be treated as an external force in the lattice Boltzmann implementation [36].

\subsection{Lattice Boltzmann implementation}

With Eq.(2.11), the hydrodynamic equations (2.7)-(2.9) can be solved using a lattice Boltzmann (LB) algorithm. The basic idea behind LB algorithm is to utilize the particle distribution functions (PDFs), discretized in time and space according to a set of lattice velocity vectors $\vec{e}_{i}$. For a two-dimensional 9-velocity model (D2Q9), the lattice velocities are chosen to be $\vec{e}_{0}=(0,0), \vec{e}_{1,3}=( \pm c, 0), \vec{e}_{2,4}=(0, \pm c), \vec{e}_{5,7}=( \pm c, \pm c)$ and $\vec{e}_{6,8}=(\mp c, \pm c)$. The lattice speed $c$ is defined by $c=\delta_{x} / \delta_{t}$, where $\delta_{x}$ is the lattice distance, and $\delta_{t}$ is the time step. The speed of sound $c_{s}$ can be related to $c$ by $c_{s}=c / \sqrt{3}$. 
To describe a binary fluid, we define two PDFs $f_{i}(\vec{x}, t)$ and $g_{i}(\vec{x}, t)$ on each lattice point. The macroscopic variables are related to the PDFs by

$$
\phi(\vec{x}, t)=\sum_{i} g_{i}(\vec{x}, t), \quad \rho(\vec{x}, t)=\sum_{i} f_{i}(\vec{x}, t), \quad \rho \vec{u}(\vec{x}, t)=\sum_{i} f_{i}(\vec{x}, t) \vec{e}_{i}+\vec{F}_{s} \delta_{t} / 2,
$$

where $\vec{F}_{s}=\mu \nabla \phi$ is the interfacial force. The time evolution equations for the PDFs, using the standard BGK (Bhatnagar-Gross-Krook) approximation, can be written as

$$
\begin{gathered}
f_{i}\left(\vec{x}+\vec{e}_{i} \delta_{t}, t+\delta_{t}\right)-f_{i}(\vec{x}, t)=\frac{1}{\tau_{f}}\left[f_{i}^{e q}(\vec{x}, t)-f_{i}(\vec{x}, t)\right]+F_{i}, \\
g_{i}\left(\vec{x}+\vec{e}_{i} \delta_{t}, t+\delta_{t}\right)-g_{i}(\vec{x}, t)=\frac{1}{\tau_{g}}\left[g_{i}^{e q}(\vec{x}, t)-g_{i}(\vec{x}, t)\right],
\end{gathered}
$$

where $f_{i}^{e q}$ and $g_{i}^{e q}$ are the equilibrium distribution functions of $f_{i}$ and $g_{i}$, defined as a power series in the velocity, $\tau_{f}$ and $\tau_{g}$ are two independent relaxation parameters, and $F_{i}$ represents the interfacial force components in the lattice space.

The governing physics of LB schemes are determined through the hydrodynamic moments of the equilibrium distribution functions and the forcing terms (i.e. the interfacial force components). The moments of $f_{i}^{e q}, g_{i}^{e q}$ and $F_{i}$ are:

$$
\begin{aligned}
& \sum_{i} f_{i}^{e q}=\rho, \quad \sum_{i} f_{i}^{e q} \vec{e}_{i}=\rho \vec{u}, \quad \sum_{i} f_{i}^{e q} \vec{e}_{i} \vec{e}_{i}=\rho \vec{u} \vec{u}+p \boldsymbol{I}, \\
& \sum_{i} g_{i}^{e q}=\phi, \quad \sum_{i} g_{i}^{e q} \vec{e}_{i}=\phi \vec{u}, \quad \sum_{i} g_{i}^{e q} \vec{e}_{i} \vec{e}_{i}=\phi \vec{u} \vec{u}+\Gamma \mu \boldsymbol{I}, \\
& \sum_{i} F_{i}=0, \quad \sum_{i} F_{i} \vec{e}_{i}=\delta_{t}\left(1-\frac{1}{2 \tau_{f}}\right) \vec{F}_{s}, \\
& \sum_{i} F_{i} \vec{e}_{i} \vec{e}_{i}=\delta_{t}\left(1-\frac{1}{2 \tau_{f}}\right)\left(\vec{u} \vec{F}_{s}+\vec{F}_{s} \vec{u}\right),
\end{aligned}
$$

A suitable choice for $f_{i}^{e q}, g_{i}^{e q}$ and $F_{i}$ that satisfies the constraints (2.15)-(2.17) is a power series expansion in the velocity

$$
\begin{gathered}
f_{i}^{e q}=w_{i}\left[A_{i}+\rho\left(\frac{\vec{e}_{i} \cdot \vec{u}}{c_{s}^{2}}+\frac{\vec{u} \vec{u}:\left(\vec{e}_{i} \vec{e}_{i}-c_{s}^{2} \boldsymbol{I}\right)}{2 c_{s}^{4}}\right)\right], \\
g_{i}^{e q}=w_{i}\left[B_{i}+\phi\left(\frac{\vec{e}_{i} \cdot \vec{u}}{c_{s}^{2}}+\frac{\vec{u} \vec{u}:\left(\vec{e}_{i} \vec{e}_{i}-c_{s}^{2} \boldsymbol{I}\right)}{2 c_{s}^{4}}\right)\right], \\
F_{i}=\left(1-\frac{1}{2 \tau_{f}}\right) w_{i}\left[\frac{\vec{e}_{i}-\vec{u}}{c_{s}^{2}}+\frac{\vec{e}_{i} \cdot \vec{u}}{c_{s}^{4}} \vec{e}_{i}\right] \cdot \vec{F}_{s} \delta_{t},
\end{gathered}
$$

where the coefficients $A_{i}$ and $B_{i}$ are given by

$$
A_{i}= \begin{cases}p / c_{s}^{2} & (i>0) \\ {\left[\rho-\left(1-w_{0}\right) p / c_{s}^{2}\right] / w_{0}} & (i=0),\end{cases}
$$




$$
B_{i}= \begin{cases}\Gamma \mu / c_{s}^{2} & (i>0) \\ {\left[\phi-\left(1-w_{0}\right) \Gamma \mu / c_{s}^{2}\right] / w_{0}} & (i=0),\end{cases}
$$

and $w_{i}$ is the weight factor with $w_{0}=4 / 9, w_{1-4}=1 / 9$ and $w_{5-8}=1 / 36$.

Using the Chapman-Enskog expansion, the lattice Boltzmann model Eqs.(2.13) and (2.14) can recover the hydrodynamic equations Eqs.(2.7)-(2.9) in the long-wavelength and low-frequency limit with the equilibrium PDFs and forcing term given by Eqs.(2.18)(2.20). The relaxation parameters $\tau_{f}$ and $\tau_{g}$ in the LB algorithm are related to the physical variables in the hydrodynamic equations $\eta$ and $M$ by

$$
\eta=\rho c_{s}^{2} \delta_{t}\left(\tau_{f}-\frac{1}{2}\right), \quad M=\delta_{t} \Gamma\left(\tau_{g}-\frac{1}{2}\right),
$$

where $\Gamma$ is a tunable parameter that appears in the equilibrium distribution function $g_{i}^{e q}$. In the following simulations, the densities $\rho_{o}$ and $\rho_{w}$ are assumed to be equal because the density difference in the commonly-used water droplet in oil is small. We set $\tau_{g}=$ $1 /(3-\sqrt{3})$ to minimize numerical errors of the convection-diffusion scheme [37]. To account for unequal viscosities of the two phases, we define the viscosity $\eta$ as a linear function of the order parameter:

$$
\eta(\phi)=\frac{1-\phi}{2} \eta_{o}+\frac{1+\phi}{2} \eta_{w}
$$

where $\eta_{o}$ and $\eta_{w}$ are the viscosities of the oil and water phases respectively. In this way, $\eta$ automatically changes across the interface with a profile similar to the tanh function.

In the numerical solution, the interfacial thickness parameter $\xi$ is a free parameter. In order to keep a sharp phase interface, $\xi$ should be chosen to be a small value. However, if $\xi$ is too small, numerical inaccuracy and instability will occur at the interface. For a finite interface thickness, straining flows can thicken or thin the interface, which must be balanced by diffusion. Meanwhile, large diffusion will excessively damp the flow. Therefore, appropriate balance between the convection and diffusion effects at the interface is important which can be described by the Peclet number, $P e$ :

$$
P e=U L / M a,
$$

where $U$ and $L$ are the characteristic velocity and length of the system. In our simulations, we compromise to have $\xi$ of 1.5 lattice grids, so that the interface is resolved typically $4 \sim 5$ grids, and $P e$ is of $O(10) \sim O(100)$.

\subsection{Boundary conditions}

Halfway bounce-back [21] is used on the fluid-solid boundary in order to obtain noslip condition. The constant flow rate or pressure boundary condition can be imposed following Zou and He [38]. We assume that fluids are only one pure single-component at 
inlet or outlet, where the unknown $g_{i}$ can be obtained by using the method proposed by Hao and Cheng [39]. For example, if the inlet boundary is assumed to be perpendicular to the $y$-direction with the lattice velocities $e_{1}, e_{5}$ and $e_{8}$ pointing into the computation domain, the PDFs $g_{1}, g_{5}$ and $g_{8}$ are unknown after the streaming step. In order to ensure a prescribed order parameter $\phi_{\text {in }}$ at inlet, these unknown PDFs must satisfy

$$
g_{1}+g_{5}+g_{8}=\phi_{i n}-\left(g_{0}+g_{2}+g_{3}+g_{4}+g_{6}+g_{7}\right) .
$$

Assuming that $g_{i}(i=1,5,8)$ are distributed by their weight factors $w_{i}$, we have

$$
g_{i}=\frac{w_{i} \phi^{*}}{w_{1}+w_{5}+w_{8}} \quad i=1,5,8
$$

with $\phi^{*}$ being the right-hand side of Eq.(2.26).

Here, the flow is symmetric due to small Reynolds number, so that the simulations are carried out only in the upper half of the domain. To incorporate the fluid-solid interactions, the wetting boundary condition proposed by van der Graaf et al. [15] is adopted in the present study. In this method, the wall is assumed to be a mixture of two fluids, thus having a certain value of the order parameter $\phi_{s}$. The derivatives of order parameter at the surface boundary can therefore be calculated using ( 9 points) compact finite difference stencils [40]. Consequently, the chemical potential and the interfacial force term in Eq. (2.20) become dependent on the properties of the neighboring solid lattice sites, resulting in a special case of the Cahn boundary condition [29]. For a desired static contact angle $\theta_{s}$, we use the following formula to assign the order parameter $\phi_{s}$ to the solid lattice sites next to the wall.

$$
\cos \left(\theta_{s}\right)=\frac{1}{2} \phi_{s}\left(3-\phi_{s}^{2}\right)
$$

where $\theta_{s}$ is related to the interfacial tensions by the Young's equation:

$$
\cos \left(\theta_{s}\right)=\frac{\sigma_{o s}-\sigma_{w s}}{\sigma}
$$

where $\sigma_{o s}$ or $\sigma_{w s}$ is the interfacial tension between the fluid phase (oil or water) and the solid surface.

\section{Validation of the present model}

\subsection{Stationary droplet flow}

The problem represents a traditional benchmark of two phase flow models. It consists of a circular droplet initially located at the centre of the lattice domain with $120 \times 120$ lattices in the $x y$-plane. The periodic boundary conditions are imposed at all boundaries. According to the Laplace's law, when the system reaches the equilibrium state, the 


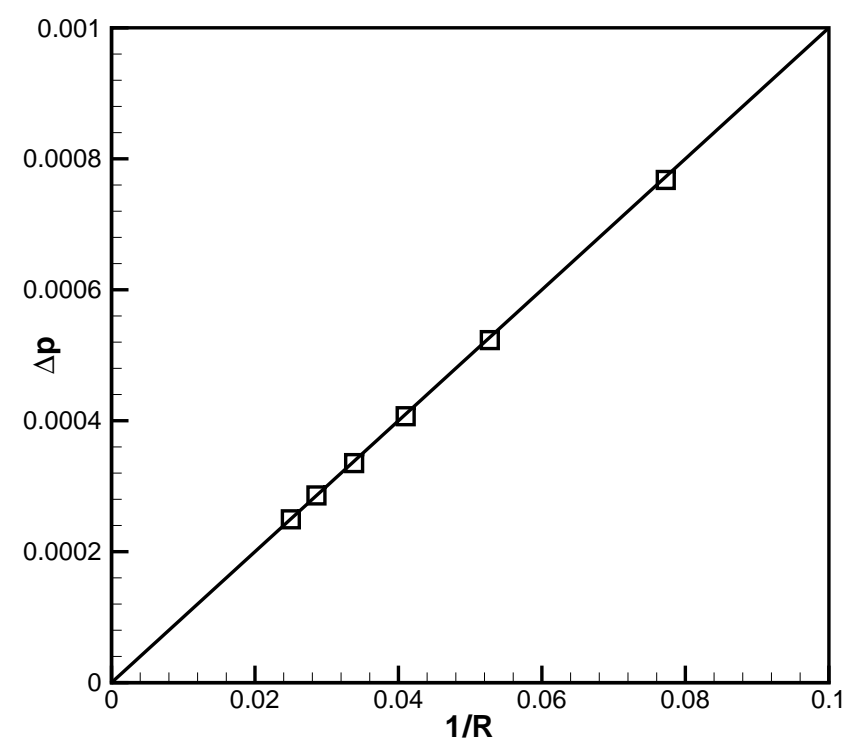

Figure 1: Comparison of the LBM results $(\square)$ with the Laplace's law (the solid line) for pressure jump across a stationary droplet interface.

pressure difference between the interior and exterior of the droplet $\Delta p$ is related to the interfacial tension $\sigma$ as

$$
\Delta p=\frac{\sigma}{R}
$$

where $R$ is the radius of the droplet. Fig. 1 shows the pressure difference $\Delta p$ against $1 / R$ using the following parameters: $\tau_{f}=1.0, \sigma=0.01$, and $\Gamma=4.0$. It can be found that the model predictions are in excellent agreement with the Laplace's law. Eq. (2.3) can also be used to verify the accuracy of the numerical results, which can be written as

$$
\phi(x, y)=\tanh \frac{R-\sqrt{\left(x-x_{0}\right)^{2}+\left(y-y_{0}\right)^{2}}}{\xi}
$$

where $x_{0}$ and $y_{0}$ are the coordinates of the centre of the droplet. Fig. 2 displays the order parameter as a function of the distance from the droplet centre, which is in good agreement with the theoretical equilibrium profile given by Eq. (3.2). This shows that our method can correctly model and capture phase interface. However, a numerical artifact observed in many numerical methods is the presence of spurious velocities at the phase interface. This is also true in our case. Fig. 3 shows the velocity vector plots in the final stage of droplet evolution for the improved color-function model presented by Lishchuk et al. [41] and the present model, where the values of $\vec{u}$ are magnified by $1.5 \times 10^{5}$ times in (a) and $1 \times 10^{7}$ times in (b) respectively. Although the improved color-function model 


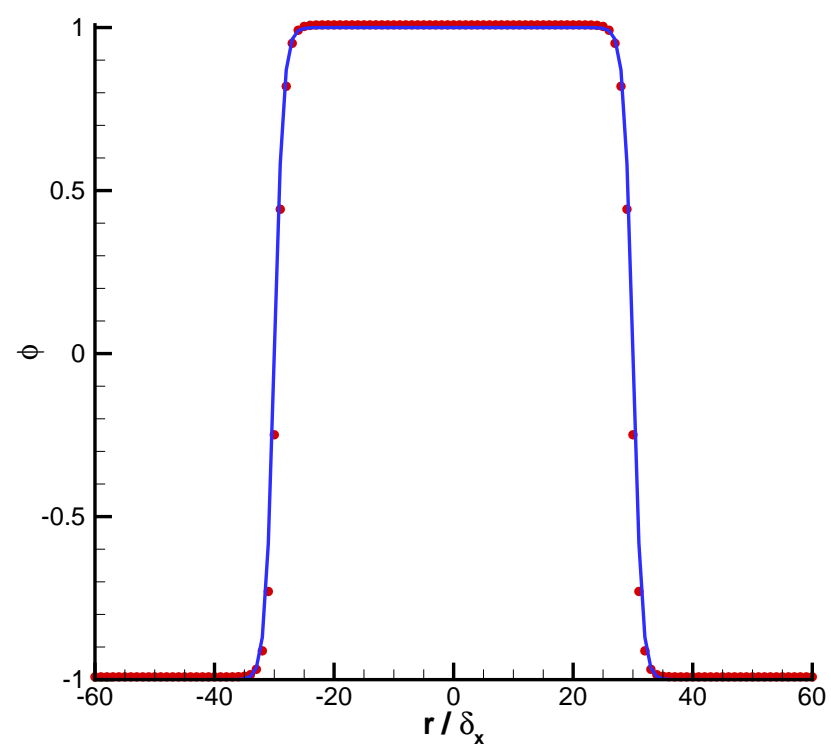

Figure 2: The profile of order parameter along the cross section of a droplet with $R=30 \delta_{x}$. The discrete symbols represent the simulation results of the present LB model and the solid line is the theoretical profile given by Eq. (3.2).

has shown to significantly reduce the spurious velocities in comparison with the Gunstensen's model [42] and the Shan-Chen model [43], it can be observed that the magnitude of maximal spurious velocity of the present model $\left(1.6 \times 10^{-6}\right)$ is much smaller than that of the improved color-function model $\left(5.2 \times 10^{-5}\right)$.

\subsection{Droplet deformation and breakup under simple shear flow}

Taylor deformation is often used to assess whether a multiphase model is able to simulate dynamic problems. A droplet is placed between two parallel plates which are moving in opposite directions to obtain linear shear in the Stokes regime (small Reynolds number), and droplet deformation is studied as a function of the shear rate (expressed as the capillary number). The definitions of the Reynolds number and capillary number are given as

$$
R e=\frac{\gamma R^{2} \rho}{\eta}, \quad C a=\frac{\gamma R \eta}{\sigma}
$$

where $\gamma=2 U / H$ is the shear rate with $U$ being the velocity of moving plate, and $H$ being the channel height. $R$ is the initial radius of the droplet. For this case, we assume that the densities and viscosities are the same for both fluids. The simulations are run at $R e=0.1$ for a droplet with the radius of 32 lattice cells in a system of $256 \times 128$ lattice cells. At the 


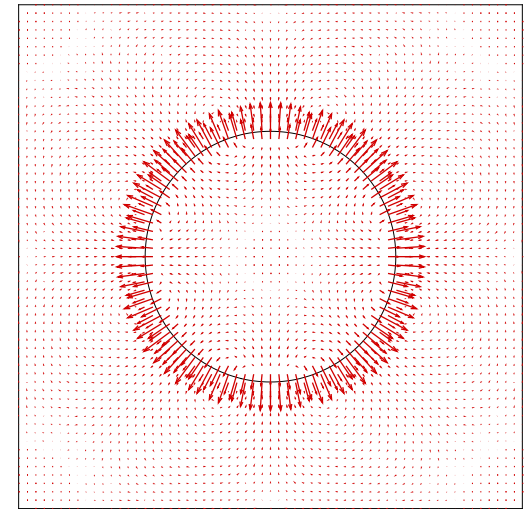

(a) Lishchuk's model

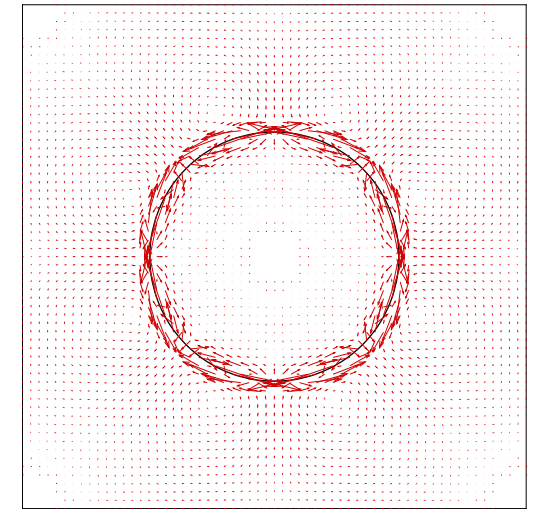

(b) Present model

Figure 3: Comparison of the spurious velocities of a droplet in stationary fluid with $\sigma=0.01$ and $R=30 \delta_{x}$ for (a) the model proposed by Lishchuk et al. [41] with the magnification rate of $1: 1.5 \times 10^{5}$ and (b) the present model with the magnification rate of $1: 1.5 \times 10^{7}$.

steady state, the droplet is assumed to be an elliptic shape, which is usually characterized by the deformation parameter $D f$, defined as

$$
D f=\frac{L-B}{L+B}
$$

where $L$ and $B$ are the major and minor axis of the ellipse. In $2 \mathrm{D}$, it is expected that $D f$ follows the Taylor relation for small $\mathrm{Ca}$ [44], which reads:

$$
D f=f(\lambda) C a,
$$

where $\lambda$ is the viscosity ratio between droplet and matrix fluid. We also carry out the simulations using the LB free energy model developed by Swift et al. [45] with the same parameter settings, as well as using VOF method with the same physical parameters and grid size. In Fig. 4 we have plotted $D f$ versus $C a$ for both LBM and VOF simulations. We observe that the simulation results of the present model are almost the same as those of Swift's model, and the results of the present model are slightly closer to the VOF results. In addition, the linear dependence of $D f$ on $C a$, i.e. Eq. (3.5), is confirmed at low capillary numbers, where $D f=1.5 \mathrm{C} a$ is obtained based on the simulation results of the present model. Fig. 5 shows the steady state droplet shapes for various capillary numbers. Qualitatively, the profiles agree well with those profiles presented in Zhou and Pozrikidis [46]: all profiles cross two points. Finally, we find that the droplet breaks up at the critical capillary number $C a_{c r}$ between 0.9 and 1.0 for $R e=1.0$, which is in agreement with the 


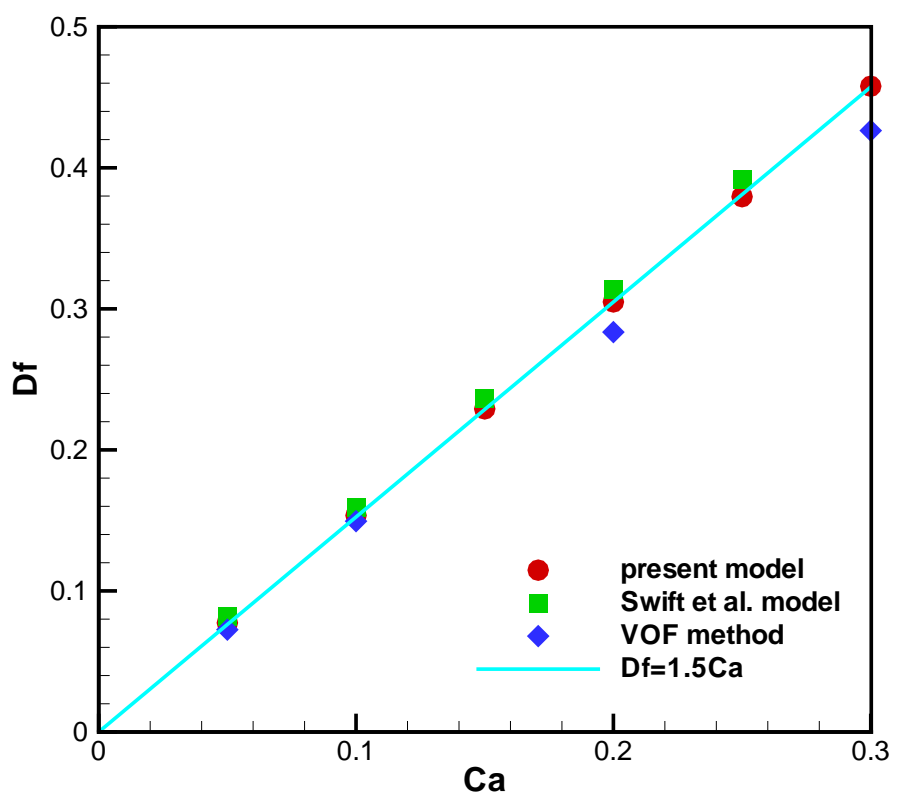

Figure 4: Taylor deformation parameter $D f$ as a function of the capillary number.

finding of Zhou and Pozrikidis [46]. Fig. 6 shows the comparison of the droplet evolution under simple shear flow for (a) $C a=0.9$ and (b) $C a=1.0$. The dimensionless time is defined as $T=\gamma t$. We notice that at $C a=0.9$ the droplet will shrink once the maximal deformation is not enough to "pinch-off" the droplet. Also, the intermediate filament will shrink when the daughter droplets detach from the bulk at $\mathrm{Ca}=1.0$. These indicate that the droplet behaves elastically to some extent.

\section{Results and discussion}

The schematic diagram of a cross-junction microchannel used in this study is shown in Fig. 7. The microchannel consists of the main channel with width $w_{c}=200 \mu \mathrm{m}$ and the two lateral channels with the same width i.e. $w_{d}=100 \mu \mathrm{m}$. The dispersed phase water is introduced at the inlet of main channel, and the continuous phase oil is injected into the lateral channels. We aim to investigate the droplet formation mechanism in a microfluidic cross-junction. To quantitatively understand the underlying flow physics, we need 3D simulations to resolve intermolecular interactions at the interface and their effect on the hydrodynamics. However, to accurately resolve interface with typical thickness of $1 \mathrm{~nm}$ and surrounding fluid flowfield, it is still not practical with today's computer technology. Therefore, we run our simulations in $2 \mathrm{D}$ to understand flow physics qualitatively. The 


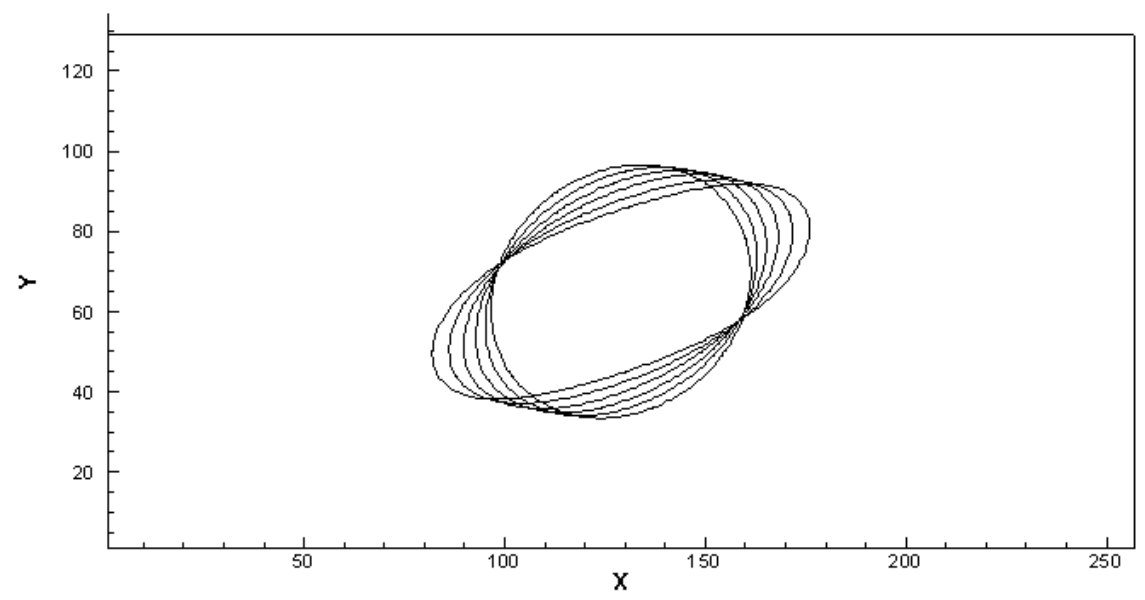

Figure 5: Stable droplet shape depicted as contour lines of $\phi=0$, for various capillary numbers: $C a=$ $\{0.05,0.1,0.15,0.2,0.25,0.3\}$.

computational domain consists of $360 \times 30$ lattices, where only one half domain is used in the $y$-direction due to the symmetry of the problem. In all the cases, we find that mesh refinement will lead to result variations no more than $5 \%$.

For simplicity, we assume that the both fluids have the same densities, which are given by $\rho$. It is expected that this choice has negligible influence on the results, since in typical oil-water microfluidic system buoyancy-driven velocities are much smaller than the actual flow velocities. We also assume the contact angle $\theta_{s}=180^{\circ}$ so that the continuous phase fluid completely wets the walls, and the dispersed phase fluid is non-wetting. For the selected geometrical parameters $w_{c}$ and $w_{d}$, the dynamical response of fluids in a microfluidic cross-junction can be fully described by the experimentally measurable parameters including the interfacial tension $\sigma$, the inlet volumetric flow rates $\left(Q_{c}\right.$ and $\left.Q_{d}\right)$, the fluid viscosities $\left(\eta_{c}\right.$ and $\left.\eta_{d}\right)$ and density $\rho$, where the subscripts ' $c$ ' and 'd' denote the continuous and dispersed phases respectively. Based on these six parameters, the size of droplets formed in this microchannel can be defined by four dimensionless numbers following the Buckingham's Pi theorem, i.e.

$$
d=f(C a, \operatorname{Re}, Q, \lambda),
$$

where $d$ is the droplet diameter, which is defined by the diameter of a spherical droplet with the same volume as a non-spherical droplet; $\mathrm{Ca}$ is the capillary number describing relative importance of the viscosity and the interfacial tension, and it is defined by the average inlet velocity $u_{c}$ and the viscosity $\eta_{c}$ of the continuous phase, and the interfacial tension $\sigma$ as $C a=\frac{u_{c} \eta_{c}}{\sigma}=\frac{Q_{c} \eta_{c}}{2 \sigma w_{c}} ; R e$ is the Reynolds number describing the ratio of inertia to viscous stresses, i.e. $R e=\frac{\rho u_{c} w_{c}}{\eta_{c}}=\frac{\rho Q_{c}}{2 \eta_{c}} ; Q=\frac{Q_{d}}{Q_{c}}$ is the ratio of flow rates, and $\lambda=\frac{\eta_{d}}{\eta_{c}}$ is the viscosity ratio. For the flow regimes under consideration, the Reynolds number is small 

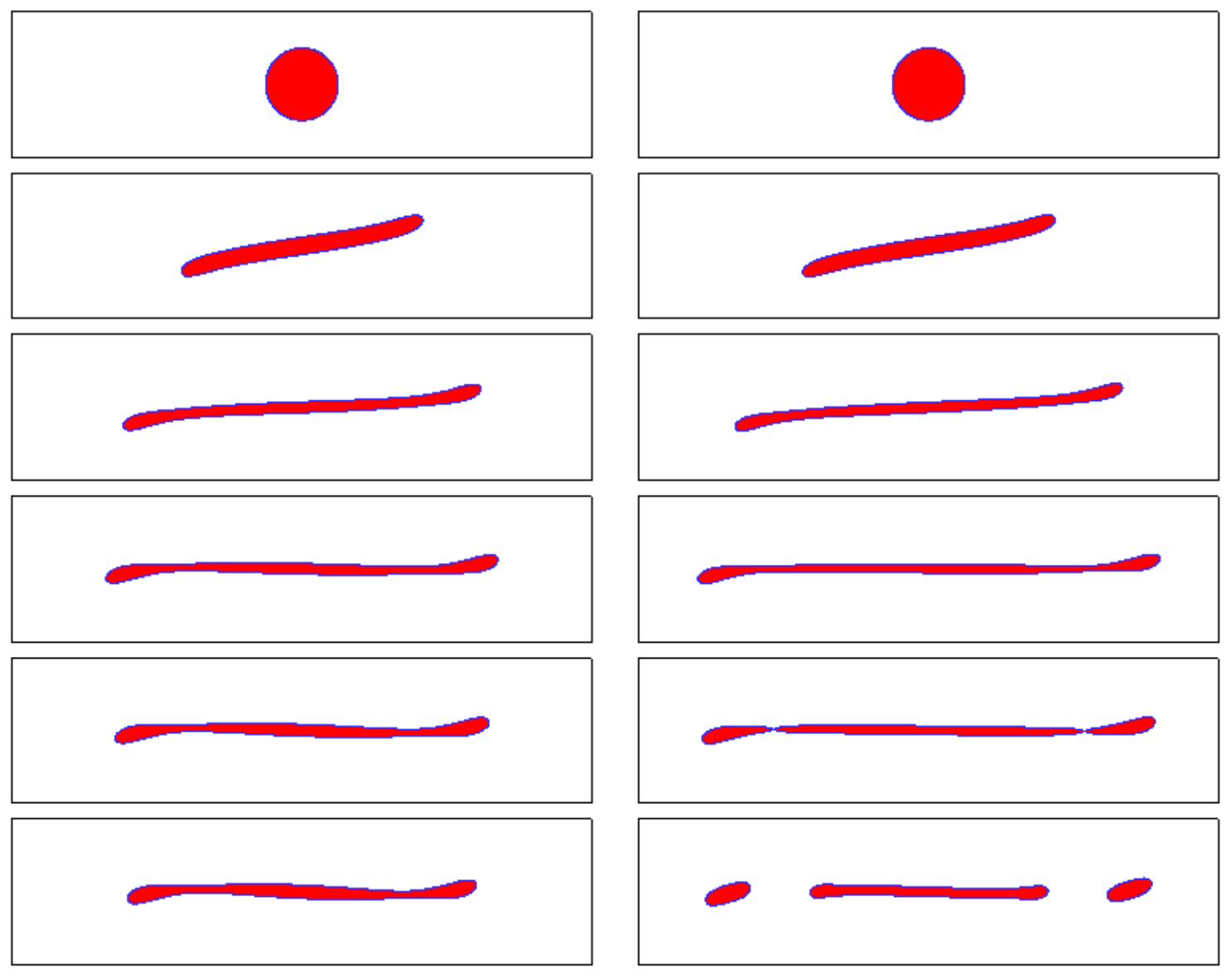

(a) $\mathrm{Ca}=0.9$

(b) $\mathrm{Ca}=1.0$

Figure 6: Snapshots of the droplet evolution under simple shear flow at $\lambda=1, \operatorname{Re}=1.0$ for (a) $\mathrm{C} a=0.9$ and (b) $C a=1.0$, taken at times $T=\{0,6,12,24,27,30\}$. 


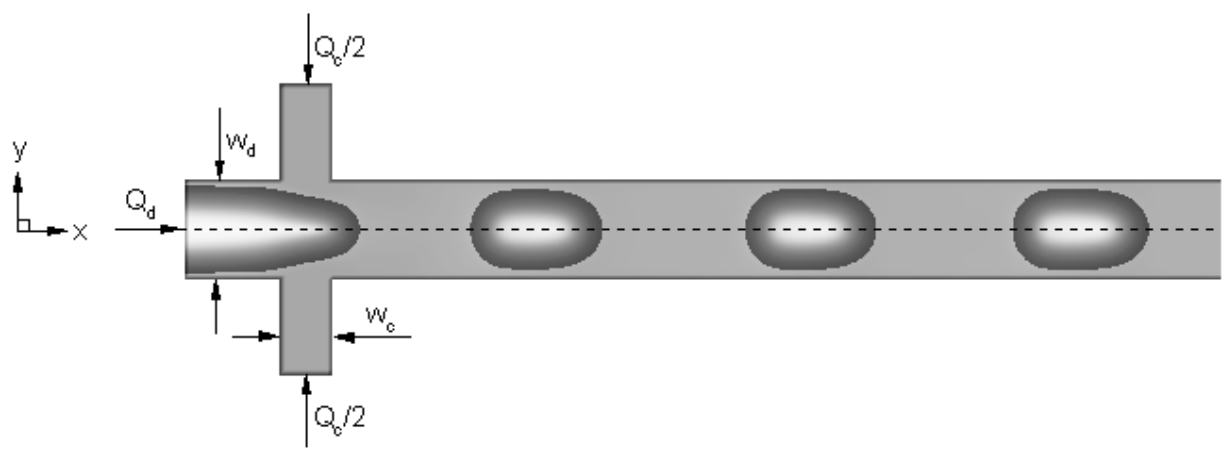

Figure 7: The schematic diagram of droplet formation in a cross-junction microchannel, where $w_{c}, w_{d}$ are the widths of the main and lateral channels, and $Q_{c}$ and $Q_{d}$ are the inlet volumetric flow rates of the continuous and dispersed phases.

$(\operatorname{Re}<1)$, and it has a negligible effect on the formation of the droplet in microchannel, so that the number of governing parameters reduces to $C a, Q$ and $\lambda$.

Different kinds of droplets, namely slug droplet, isolate droplet and satellite droplet, can be generated in the cross-junction microfluidic device, which strongly depend on the flow conditions [10]. In the present study, we focus on formation of slugs and isolate droplets, and examine the roles of the above dimensionless numbers in droplet formation.

\subsection{The effect of capillary number}

Fig. 8 shows the influence of capillary number on the droplet diameter where the interfacial tension $\sigma=0.016$, the viscosity of the continuous phase $\eta_{c}=0.08$ and the viscosity ratio $\lambda=1 / 4$. The densities of both fluids are assumed to be unity. In order to compare our simulation results with the experimental observations [19], we choose $Q_{d}=0.002,0.004$ and 0.008 in the simulations. For convenience, all quantities are expressed in the lattice units except the droplet diameter, which is in the physical unit. To match these LB simulation parameters to their physical values, we choose three reference quantities: a length scale $L_{0}$, a time scale $T_{0}$, and a mass scale $M_{0}$. In this study, $L_{0}=10^{-5} \mathrm{~m}, T_{0}=10^{-6} \mathrm{~s}$ and $M_{0}=10^{-12} \mathrm{~kg} / \mathrm{m}^{3}$. A simulation parameter with dimensions $[L]^{n 1}[T]^{n 2}[M]^{n 3}$ is multiplied by $\left[L_{0}\right]^{n 1}\left[T_{0}\right]^{n 2}\left[M_{0}\right]^{n 3}$ to obtain the physical value. Following this criterion, for example, we can obtain the physical value of interfacial tension $\sigma^{\text {phy }}$ by: $\sigma^{\text {phy }}=\sigma \frac{M_{0}}{T_{0}^{3}}=0.016 \frac{10^{-12}}{\left(10^{-6}\right)^{2}}=0.016 \mathrm{~N} \mathrm{~m}^{-1}$, and the physical value of continuous phase viscosity $\eta_{c}^{p h y}$ by: $\eta_{c}^{p h y}=\eta_{c} \frac{M_{0}}{L_{0} T_{0}}=0.08 \frac{10^{-12}}{10^{-5} \cdot 10^{-6}}=8 \times 10^{-3}$ Pa s.

For each $Q_{d}$, we control Ca by only varying $Q_{c}$. At a fixed dispersed phase flow rate, the droplet diameter becomes smaller when $\mathrm{Ca}$ increases. At small $\mathrm{Ca}$, the flow rate of the dispersed phase shows a significant effect on the droplet diameter, and larger $Q_{d}$ is expected to generate a larger droplet; whereas the influence of $Q_{d}$ gradually weakens as 


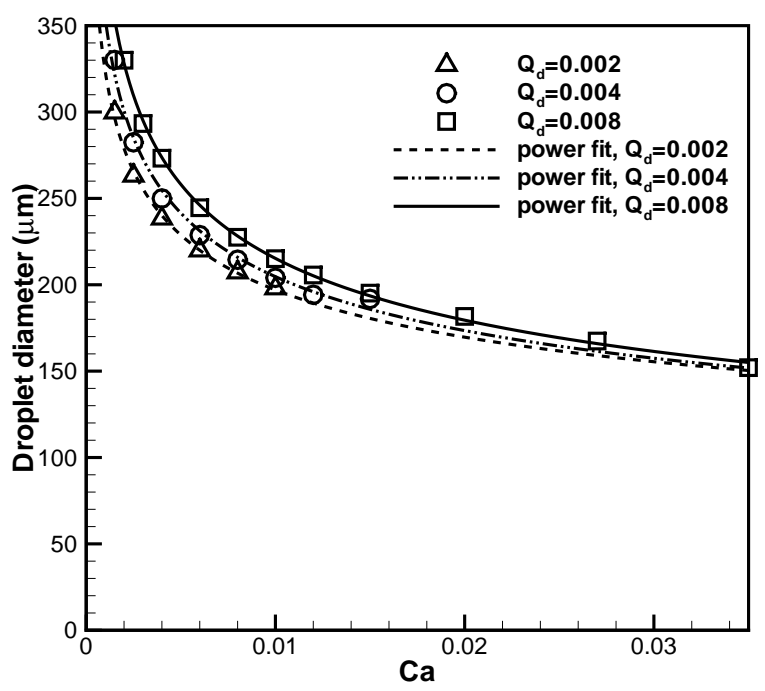

Figure 8: The influence of capillary number on the droplet diameter at the dispersed phase flow rate $Q_{d}$ of $2 \times 10^{-3}, 4 \times 10^{-3}$ and $8 \times 10^{-3}$ respectively. The lines represent the power fittings of the simulation results.

$\mathrm{Ca}$ increases. These observations are consistent with the experimental findings of Tan et al. [19]. Fig. 9 shows the snapshots of droplet formation at a fixed $Q_{d}=8 \times 10^{-3}$ for various capillary numbers. At large capillary number, the dispersed phase fluid breaks up easily leading to small droplets. When the capillary number increases, the distance between two neighboring droplets increases.

\subsection{The effect of flow rate ratio}

The effect of flow rate ratio on droplet formation is investigated over a wide range of capillary numbers. We still keep the fluid pair fixed, where the interfacial tension is now
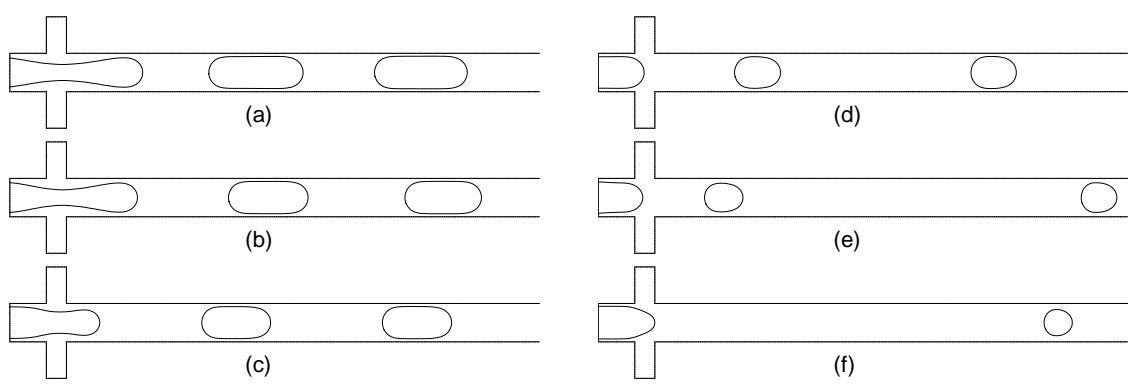

Figure 9: The droplet generation at the dispersed phase flow rate $Q_{d}=8 \times 10^{-3}$ for various capillary numbers: (a) 0.002; (b) 0.003; (c) 0.004; (d) 0.01; (e) 0.02; and (f) 0.035 . 

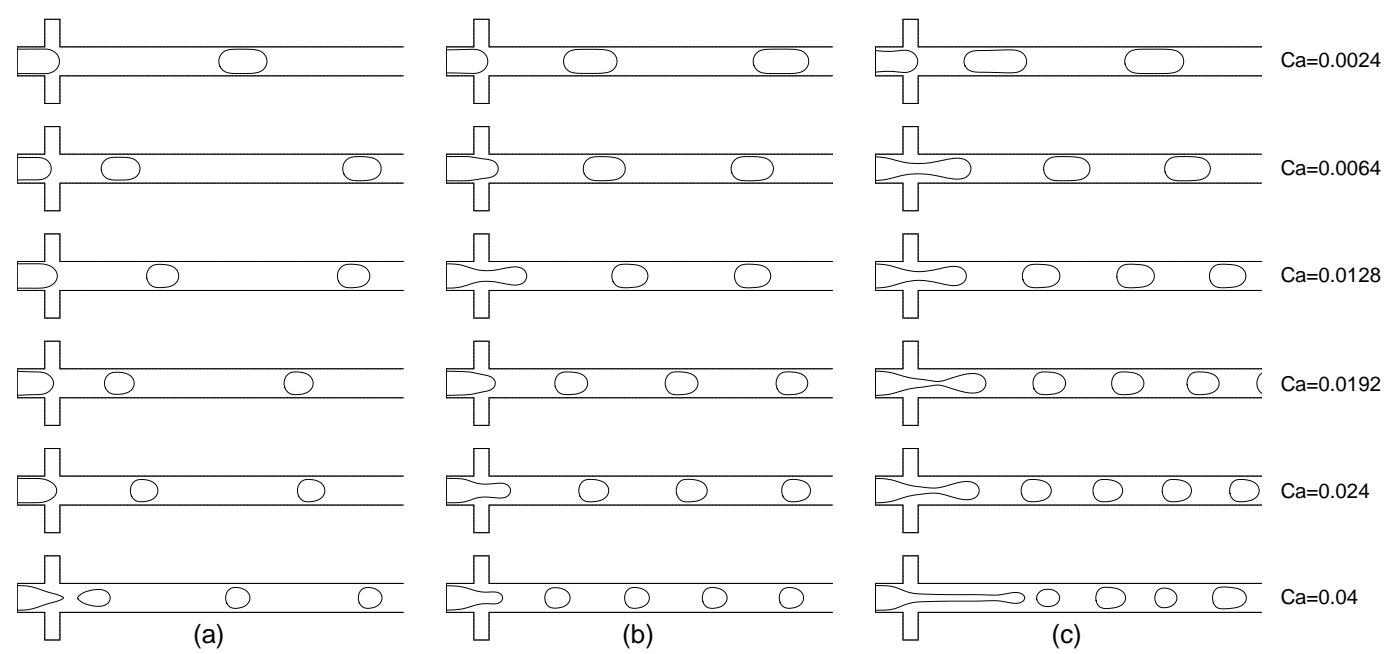

Figure 10: The flow patterns for various capillary numbers and the flow rate ratios at a fixed viscosity ratio $\lambda=1 / 4$ : (a) $Q=1 / 6$; (b) $Q=1 / 3$; and (c) $Q=1 / 2$. Each row uses the same capillary number, which is labelled on the right side.

set to be 0.01 (the corresponding physical value is $0.01 \mathrm{~N} \mathrm{~m}^{-1}$ ), and the densities and viscosities of both fluids are kept the same as in the previous section. Three different flow rate ratios, i.e. $Q=1 / 6,1 / 3$, and $1 / 2$, are used in the simulations. For each flow rate ratio, the capillary number varies from 0.0024 to 0.04 , typically found in microfluidic droplet generation. To keep the flow rate ratio fixed, both $Q_{c}$ and $Q_{d}$ must vary as $C a$ varies.

As shown in Fig. 10, the monodisperse droplets are regularly generated for all capillary numbers at low flow rate ratios, i.e. $Q=1 / 6,1 / 3$. For the high flow rate ratio $Q=1 / 2$, the highly uniform droplets can be generated at small capillary number i.e. $C a \leq 0.024$. For high capillary number $C a=0.04$, the generated droplets are not uniform, where a long dispersed jet is observed with the detachment point moving progressively downstream. In the future, we will investigate this jetting mode in detail. For all of the cases with uniform droplets generated, the distance between two neighboring droplets and their size decrease when $\mathrm{Ca}$ increases at the same flow rate ratio $Q$. Increasing the flow rate ratio can decrease the distance between two neighboring droplets at the same $\mathrm{Ca}$.

Fig. 11 shows the predicted droplet diameter as a function of capillary number for three different flow rate ratios. The droplets grow as the flow rate ratio increases but they become smaller as the capillary number increases. This trend is consistent with the experimental observations for the droplet production in a variety of geometries [47]. Two distinguished regimes are identified and divided by a critical capillary number $\left(C a_{c r}\right)$, which is 0.01 in our simulations. For low $C a$, i.e. $C a \leq C a_{c r}$, the flow rate can significantly affect the droplet size, and the droplet diameter $d$ exhibits a power-law dependence on the capillary number, i.e. $d \propto C a^{-0.147}$, where the power-law exponent $\alpha=-0.147$ is independent of the flow rate $Q$. This finding was also experimentally observed by Tan et 


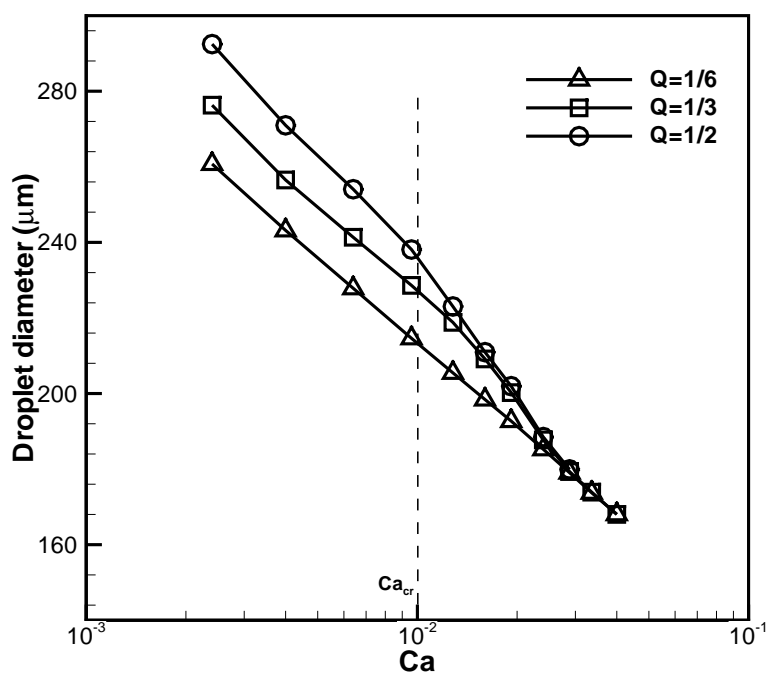

Figure 11: The effect of capillary number and flow rate ratio on droplet diameter at a fixed viscosity ratio, $\lambda=1 / 4$. Note that, for $Q=1 / 2$ and $C a>0.024$, the measured droplet diameters are not plotted in this figure due to irregular droplets are generated.

al. [19] for the plug generation in a cross-junction microchannel. Obviously, the formation of plugs in cross-junction is different from the experimental observation of Garstecki et al. [14] in microfluidic T-junctions where the plug size depends predominantly on the flow rate ratio $Q$ rather than the capillary number $C a$. Garstecki et al. [14] concluded that the droplet breakup is completely dominated by the squeezing pressure, which arises when the emerging droplet obstructs the continuous phase stream in the main channel. However, a recent numerical study [48] on the plugs formed in T-junctions observed that the droplet size is also dependent on $\mathrm{Ca}$ at low capillary numbers. In addition, the 3D cross-junction simulation of $\mathrm{Wu}$ et al. [25] showed that, at a fixed flow rate ratio, the droplet size has no sign of approaching a constant value as the capillary number decreases, although they did not report the power-law dependence of droplet size on the capillary number. In the next section, we will also show that the viscous force plays an important role in the process of droplet generation in this regime, which we call the squeezing-like regime in this paper. When $\mathrm{Ca}>\mathrm{Ca} a_{c r}$, the dependence of the droplet diameter on the capillary number does not exhibit a fixed power-law behavior, and the effect of flow ratio diminishes quickly as $\mathrm{Ca}$ increases. More specifically, the droplet diameter is completely independent of the flow rate ratio when $C a \geq 0.024$. This feature, in the dripping regime, is similar to the T-junctions $[27,49]$. In the dripping regime, the viscous force is significant, together with the capillary force, determine the process of droplet breakup, which is significantly different from the droplet generation in unbound flow conditions due to the confinement of channel walls [49]. 


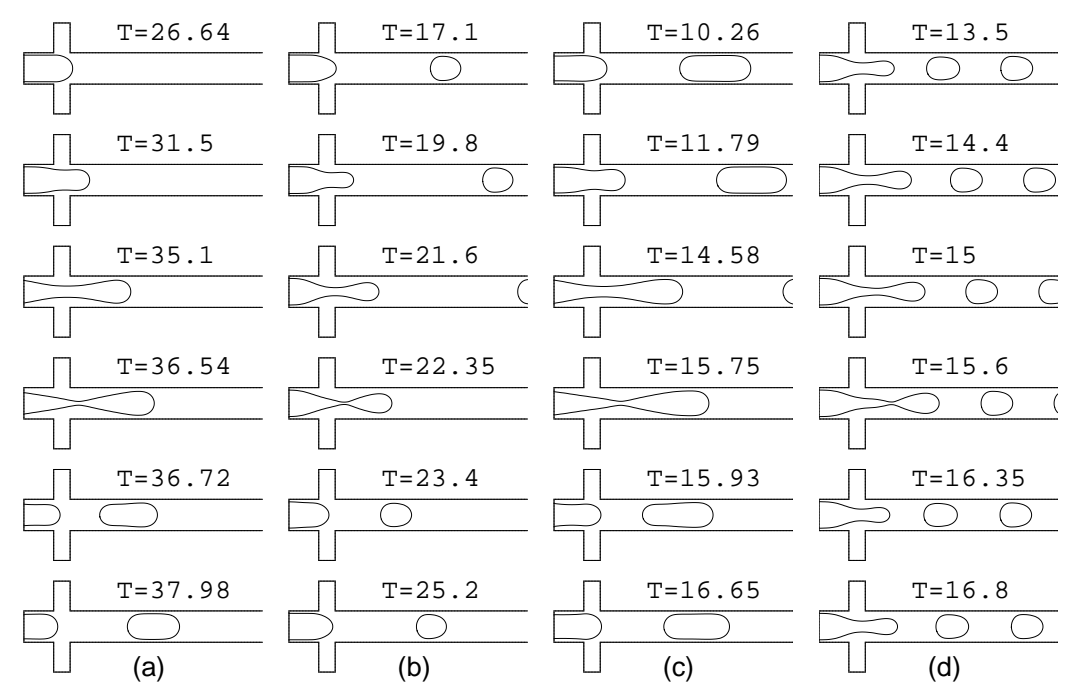

Figure 12: A series of instantaneous states of droplet formation for (a) $Q=1 / 6, C a=0.0024$; (b) $Q=1 / 6, C a=$ 0.024 ; (c) $Q=1 / 2, C a=0.0024$ and (d) $Q=1 / 2, C a=0.024$. The viscosity ratio $\lambda=1 / 4$, and the dimensionless time is defined as $T=\frac{u_{c}}{w_{c}} t$.

Fig. 12 shows a series of instantaneous states of droplet formation with four pairs of $Q$ and Ca: (a) $Q=1 / 6, C a=0.0024$; (b) $Q=1 / 6, C a=0.024$; (c) $Q=1 / 2, C a=0.0024$ and (d) $Q=1 / 2, C a=0.024$. It can be seen that the droplet formation process has three stages, i.e. expansion (i)-(iii), necking (iv) and figuration (v)-(vi). At small capillary number i.e. $C a=0.0024$, the incoming dispersed phase fluid tends to occupy the full width of the outlet channel and the dispersed phase interface has a large deviation from the solid wall at the main channel entrance, so the squeezing pressure is pronounced when the emerging droplet obstructs the channel. While in the dripping regime i.e. $\mathrm{C} a=0.024$, the dispersed phase fluid occupies only part of the outlet channel, and smaller droplets are formed. At small $Q$, i.e. $Q=1 / 6$, the droplets are pinched off close to the junction corner for all the capillary numbers. Increasing $Q$ will move the detachment point further to the downstream. At small $Q$ (see Fig. 11), the two regimes i.e. the squeezing-like and dripping regimes are not distinguishable, which was also observed experimentally in droplet generation in a T-junction by Christopher et al. [16]. Fig. 12 also gives the dimensionless times corresponding to various instantaneous states of droplet formation process. The dimensionless time is taken as $T=\frac{u_{c}}{w_{c}} t=\frac{Q_{c}}{2 w_{c}^{2}} t$. It can be clearly seen that the increase in $Q$ (or $\mathrm{Ca}$ ) can lead to the decrease in elapsed dimensionless time for generating each droplet.

\subsection{The effect of viscosity ratio}

The viscosity ratio is known to affect breakup of isolated droplets [50] and liquid jets [51]. Moreover, the numerical and experimental investigations on T-junction microchannel re- 


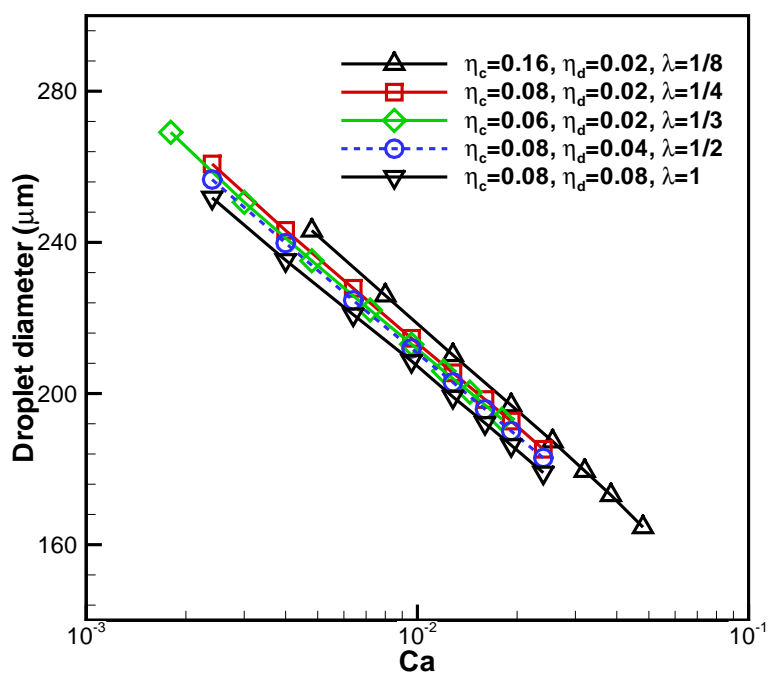

Figure 13: The effect of capillary number and viscosity ratio on droplet diameter at a fixed flow rate ratio, i.e. $Q=1 / 6$.

veal that the effect of viscosity ratio is most pronounced in the dripping regime and diminishes in the squeezing regime $[13,14,27,49]$. However, in a cross-junction with oilwater two-phase flow, Tan et al. [19] found that the plug size is independent of viscosity ratio and decreases when the continuous phase viscosity increases. Christopher et al. [16] experimentally observed in the T-junction microfluidic devices that the viscosity ratio influences the droplet size only when the viscosities of two fluids are similar. When the viscosity ratio $\lambda<1 / 50$, the resulting droplet size is independent of the viscosity ratio. In addition, $\mathrm{Fu}$ et al. [10] found that the viscosity of liquid phase is one of the dominant parameters during the bubble breakup process. To understand the complex effect of viscosity ratio and viscosity on droplet breakup, we numerically simulated the droplet breakup process in a cross-junction. In the simulations, the interfacial tension is set to be 0.01 (the corresponding physical value is $0.01 \mathrm{~N} \mathrm{~m}^{-1}$ ). The flow rate of the continuous phase $Q_{c}$ varies from 0.006 to 0.06 , and the flow rate ratio $Q$ is fixed at $1 / 6$ so that the droplet breakup always occurs close to the junction as the experiment of Tan et al. [19].

Fig. 13 shows that the droplet size is found to be weakly dependent on the viscosity ratio, which is very similar to the experimental observation by Christopher et al. [16] in a T-junction where the viscosity ratio is smaller than 1/50. Fig. 14 shows the flow patterns for different viscosities of the continuous phase. The droplet size and the distance between two neighboring droplets decrease as the viscosity of the continuous phase increases at a fixed $Q_{c}\left(\right.$ or $Q_{d}$ ). The result is consistent with the experimental finding in the cross-junction by Tan et al. [19]. However, it is different from the experimental observa- 


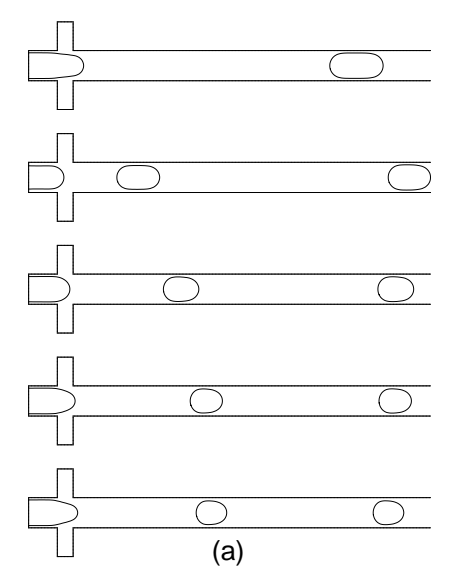

(a)

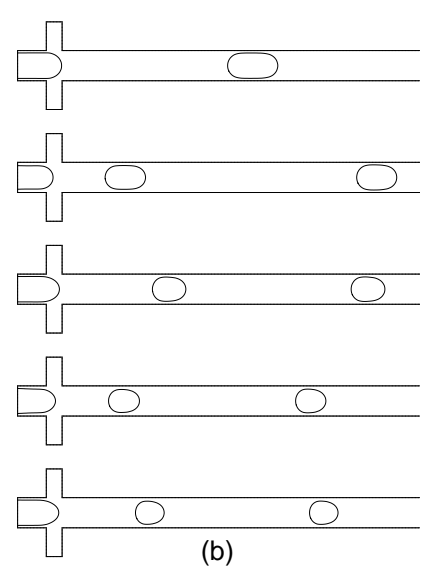

(b)

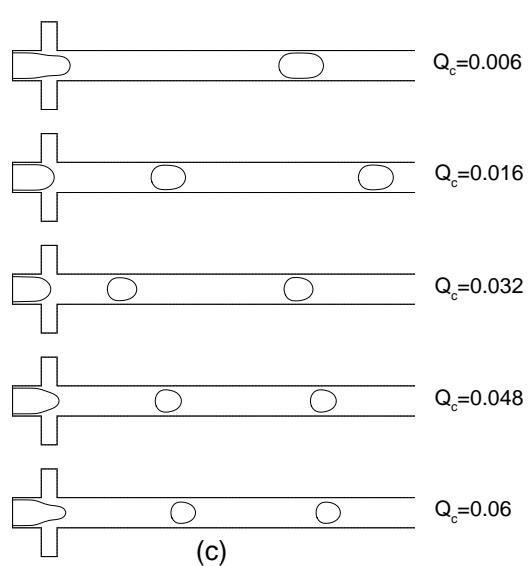

(c)

Figure 14: The flow patterns for various $\eta_{c}$ and $Q_{c}$ at a fixed flow rate ratio $Q=1 / 6$ : (a) $\eta_{c}=0.06$; (b) $\eta_{c}=0.08$; and (c) $\eta_{c}=0.16$. Each row uses the same $Q_{c}$, which is labeled on the right side.

tions on droplet formation in the T-junction microchannel $[13,14]$ and bubble formation in a cross-junction microchannel $[8,9]$, where the plug length is independent of the viscosity of the continuous phase. Tan et al. [19] argued that the difference may attribute to the symmetrical flow route of cross-junction and small capillary number in bubble formation. In addition, the sharp corner of T-junctions and the large density and viscosity ratios in the bubble formation may also be responsible.

There are two mechanisms which influence plug/droplet size. One is the dynamic breakup of interface, which can mainly be affected by the flow rate ratio $Q$. The other is the balance between the viscous force and the capillary force, which can be described by the capillary number. Tan et al. [19] proposed a scaling law to predict the plug length, which will be similarly used to estimate droplet sizes apart from the flow conditions in the dripping regime with large $Q$ where the droplet breakup occurs at further downstream of the junction. Therefore, the droplet diameter $d$ can be correlated as

$$
d=k Q^{\alpha} C a^{\beta}
$$

where $\alpha$ and $\beta$ represent the influences of two mechanisms. Our numerical simulations suggest that this scaling law works well with the coefficients of $k=129.32, \alpha=0.103$ and $\beta=-0.147$ (see Fig. 15). Although the scaling law may not be quantitatively accurate as $3 \mathrm{D}$ effects are not considered, our 2D simulation results can reveal the underlying mechanisms of droplet breakup which are consistent with the experimental findings [19].

\section{Conclusions}

Water droplet formation in a microfluidic cross-junction has been simulated using the phase-field lattice Boltzmann model. The influence of capillary number, flow rate ratio, 


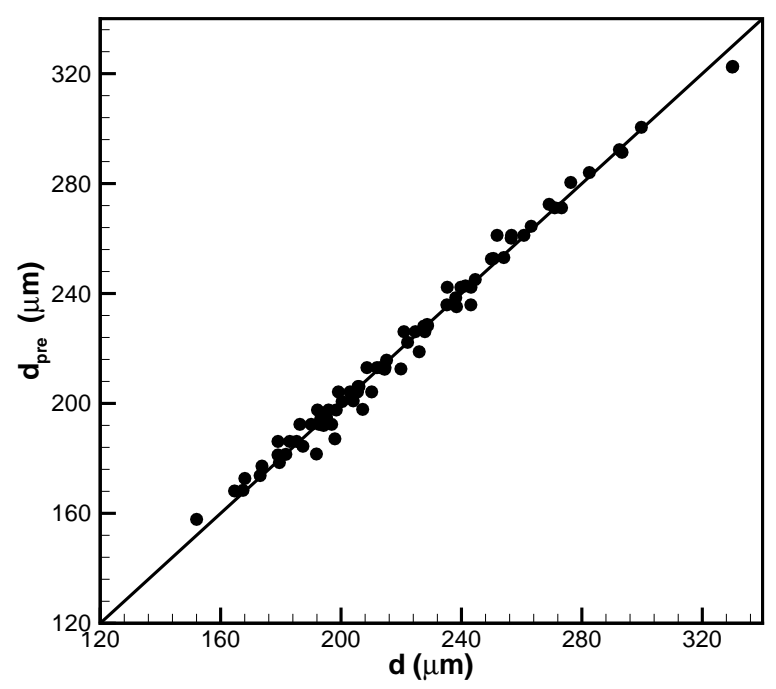

Figure 15: Comparison of droplet diameter between the fitting results $d_{\text {pre }}$ from Eq. (4.2) and the simulation results $d$.

viscosity ratio, and viscosity of continuous phase on droplet formation has been systematically studied over a wide range of capillary numbers. Two different regimes, i.e. the squeezing-like and dripping regimes, are clearly identified with the transition occurring at a critical capillary number $C a_{c r}=0.01$. However, these two regimes are difficult to distinguish when the flow rate ratio decreases. In the squeezing-like regime, i.e. $\mathrm{Ca} \leq \mathrm{Ca}$ r, the droplet breakup is influenced by not only the squeezing pressure but also the viscous force. While in the dripping regime, i.e. $\mathrm{Ca}>\mathrm{Ca} a_{c r}$, the viscous force plays an increasingly important role in the breakup process and the droplet size becomes quickly independent of the flow rate ratio for large capillary number. As the droplet size is weakly dependent on the viscosity ratio, a scaling law is constructed to estimate the generated droplet size, which depends on the capillary number and the flow rate ratio.

\section{References}

[1] H. Song, D. L. Chen, R. F. Ismagilov, Reactions in droplets in microfluidic channels, Angew. Chem. Int. Ed. 45 (2006) 7336-7356.

[2] A. J. deMello, Microfluidics: DNA amplification moves on, Nature 422 (2003) 28-29.

[3] A. Huebner, S. Sharma, M. Srisa-Art, F. Hollfelder, J. B. Edel, A. J. deMello, Microdroplets: A sea of applications?, Lab Chip 8 (2008) $1244-1254$.

[4] H. Stone, A. Stroock, A. Ajdari, Engineering flows in small devices microfluidics toward a Lab-on-a-chip, Annu. Rev. Fluid Mech. 36 (2004) 381-411.

[5] M. Yasuno, S. Sugiura, S. Iwamoto, M. Nakajima, A. Shono, K. Satoh, Monodispersed microbubble formation using microchannel technique, AIChE J. 50 (2004) 3227-3233. 
[6] S. Sugiura, M. Nakajima, M. Seki, Prediction of droplet diameter for microchannel emulsification: Prediction model for complicated microchannel geometries, Ind. Eng. Chem. Res. 43 (2004) 8233-8238.

[7] S. L. Anna, N. Bontoux, H. A. Stone, Formation of dispersions using "flow focusing" in microchannels, Appl. Phys. Lett. 82 (2003) 364-366.

[8] T. Cubaud, M. Tatineni, X. Zhong, C.-M. Ho, Bubble dispenser in microfluidic devices, Phys. Rev. E 72 (2005) 037302.

[9] P. Garstecki, H. A. Stone, G. M. Whitesides, Mechanism for flow-rate controlled breakup in confined geometries: A route to monodisperse emulsions, Phys. Rev. Lett. 94 (2005) 164501.

[10] T. Fu, Y. Ma, D. Funfschilling, H. Z. Li, Bubble formation and breakup mechanism in a microfluidic flow-focusing device, Chem. Eng. Sci. 64 (10) (2009) 2392 - 2400.

[11] T. Thorsen, R. W. Roberts, F. H. Arnold, S. R. Quake, Dynamic pattern formation in a vesiclegenerating microfluidic device, Phys. Rev. Lett. 86 (2001) 4163-4166.

[12] T. Nisisako, T. Torii, T. Higuchi, Droplet formation in a microchannel network, Lab Chip 2 (2002) 24-26.

[13] J. H. Xu, G. S. Luo, S. W. Li, G. G. Chen, Shear force induced monodisperse droplet formation in a microfluidic device by controlling wetting properties, Lab Chip 6 (2005) 131-136.

[14] P. Garstecki, M. J. Fuerstman, H. A. Stone, G. M. Whitesides, Formation of droplets and bubbles in a microfluidic T-junction-scaling and mechanism of break-up, Lab Chip 6 (2006) 437-446.

[15] S. van der Graaf, T. Nisisako, C. G. P. H. Schroën, R. G. M. van der Sman, R. M. Boom, Lattice Boltzmann simulations of droplet formation in a T-shaped microchannel, Langmuir 22 (2006) 4144-4152.

[16] G. F. Christopher, N. N. Noharuddin, J. A. Taylor, S. L. Anna, Experimental observations of the squeezing-to-dripping transition in T-shaped microfluidic junctions, Phys. Rev. E 78 (2008) 036317.

[17] P. B. Umbanhowar, V. Prasad, D. A. Weitz, Monodisperse emulsion generation via drop break off in a coflowing stream, Langmuir 16 (2000) 347-351.

[18] J. Hua, B. Zhang, J. Lou, Numerical simulation of microdroplet formation in coflowing immiscible liquids, AIChE J. 53 (2007) 2534-2548.

[19] J. Tan, J. Xu, S. Li, G. Luo, Drop dispenser in a cross-junction microfluidic device: Scaling and mechanism of break-up, Chem. Eng. J. 136 (2008) $306-311$.

[20] M. R. Davidson, D. J. E. Harvie, J. J. Cooper-White, Flow focusing in microchannels, ANZIAM J. 46 (2004) C47-C58.

[21] S. Succi, The Lattice Boltzmann Equation for Fluid Dynamics and Beyond, Oxford: Oxford University Press, 2001.

[22] S. Chen, G. D. Doolen, Lattice Boltzmann method for fluid flows, Annu. Rev. Fluid Mech. 30 (1998) 329-364.

[23] M. M. Dupin, I. Halliday, C. M. Care, Simulation of a microfluidic flow-focusing device, Phys. Rev. E 73 (2006) 055701.

[24] Z. Yu, O. Hemminger, L.-S. Fan, Experiment and lattice Boltzmann simulation of two-phase gas-liquid flows in microchannels, Chem. Eng. Sci. 62 (2007) $7172-7183$.

[25] L. Wu, M. Tsutahara, L. S. Kim, M. Ha, Three-dimensional lattice Boltzmann simulations of droplet formation in a cross-junction microchannel, Int. J. Multiphase Flow 34 (2008) 852864.

[26] L. Kim, H. Jeong, M. Ha, K. Kim, Numerical simulation of droplet formation in a microchannel using the lattice Boltzmann method, J. Mech. Sci. Technol. 22 (2008) 770-779. 
[27] H. Liu, Y. Zhang, Droplet formation in a T-shaped microfluidic junction, J. Appl. Phys. 106 (2009) 034906.

[28] A. Gupta, S. M. S. Murshed, R. Kumar, Droplet formation and stability of flows in a microfluidic T-junction, Appl. Phys. Lett. 94 (2009) 164107.

[29] J. W. Cahn, Critical point wetting, J. Chem. Phys. 66 (1977) 3667-3672.

[30] M. D. Menech, Modeling of droplet breakup in a microfluidic T-shaped junction with a phase-field model, Phys. Rev. E 73 (2006) 031505.

[31] C. Zhou, P. Yue, J. J. Feng, Formation of simple and compound drops in microfluidic devices, Phys. Fluids 18 (2006) 092105.

[32] W. Yu, C. Zhou, Coalescence of droplets in viscoelastic matrix with diffuse interface under simple shear flow, J. Polym. Sci. Part B: Polym. Phys. 45 (2007) 1856-1869.

[33] R. van der Sman, S. van der Graaf, Emulsion droplet deformation and breakup with lattice Boltzmann model, Comput. Phys. Commun. 178 (2008) 492-504.

[34] J. S. Rowlinson, B. Widom, Molecular Theory of Capillarity, London: Clarendon Press, 1989.

[35] V. M. Kendon, M. E. Cates, I. Pagonabarraga, J. C. Desplat, P. Bladon, Inertial effects in three-dimensional spinodal. decomposition of a symmetric binary fluid mixture: a lattice Boltzmann study, J. Fluid Mech. 440 (2001) 147-203.

[36] Z. Guo, C. Zheng, B. Shi, Discrete lattice effects on the forcing term in the lattice Boltzmann method, Phys. Rev. E 65 (2002) 046308.

[37] R. G. M. van der Sman, Galilean invariant lattice Boltzmann scheme for natural convection on square and rectangular lattices, Phys. Rev. E 74 (2006) 026705.

[38] Q. Zou, X. He, On pressure and velocity boundary conditions for the lattice Boltzmann BGK model, Phys. Fluids 9 (1997) 1591-1598.

[39] L. Hao, P. Cheng, Lattice Boltzmann simulations of liquid droplet dynamic behavior on a hydrophobic surface of a gas flow channel, J. Power Sources 190 (2009) $435-446$.

[40] C. M. Pooley, K. Furtado, Eliminating spurious velocities in the free-energy lattice Boltzmann method, Phys. Rev. E 77 (2008) 046702.

[41] S. V. Lishchuk, C. M. Care, I. Halliday, Lattice Boltzmann algorithm for surface tension with greatly reduced microcurrents, Phys. Rev. E 67 (2003) 036701.

[42] A. K. Gunstensen, D. H. Rothman, S. Zaleski, G. Zanetti, Lattice Boltzmann model of immiscible fluids, Phys. Rev. A 43 (8) (1991) 4320-4327.

[43] X. Shan, H. Chen, Lattice Boltzmann model for simulating flows with multiple phases and components, Phys. Rev. E 47 (3) (1993) 1815-1819.

[44] T. Roths, C. Friedrich, M. Marth, J. Honerkamp, Dynamics and rheology of the morphology of immiscible polymer blends —on modeling and simulation, Rheol. Acta 41 (3) (2002) 211222.

[45] M. R. Swift, E. Orlandini, W. R. Osborn, J. M. Yeomans, Lattice Boltzmann simulations of liquid-gas and binary fluid systems, Phys. Rev. E 54 (5) (1996) 5041-5052.

[46] H. Zhou, C. Pozrikidis, The flow of suspensions in channels: Single files of drops, Phys. Fluids A 5 (2) (1993) 311-324.

[47] G. F. Christopher, S. L. Anna, Microfluidic methods for generating continuous droplet streams, J. Phys. D: Appl. Phys. 40 (2007) R319-R336.

[48] A. Gupta, R. Kumar, Effect of geometry on droplet formation in the squeezing regime in a microfluidic T-junction, Microfluidics and Nanofluidics 8 (6) (2010) 799-812.

[49] M. D. Menech, P. Garstecki, F. Jousse, H. A. Stone, Transition from squeezing to dripping in a microfluidic T-shaped junction, J. Fluid Mech. 595 (2008) 141-161.

[50] H. A. Stone, Dynamics of drop deformation and breakup in viscous fluids, Annu. Rev. Fluid 
Mech. 26 (1994) 65-102.

[51] S. Tomotika, On the instability of a cylindrical thread of a viscous liquid surrounded by another viscous fluid, Proc. R. Soc. London, Ser. A 150 (1935) 322-337. 